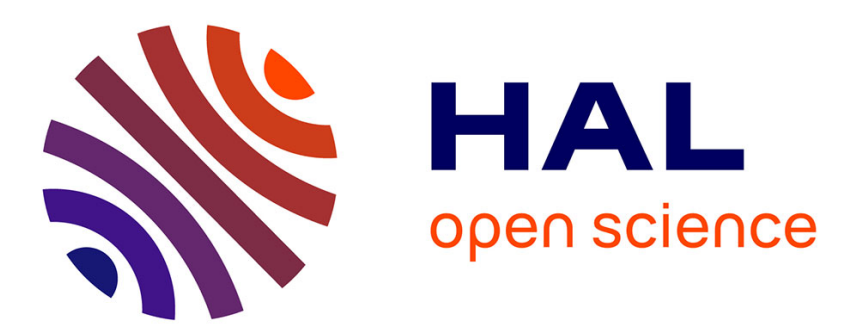

\title{
Temperature dependence of high field electromechanical coupling in ferroelectric ceramics
}

\author{
P M Weaver, M G Cain, M Stewart
}

\section{To cite this version:}

P M Weaver, M G Cain, M Stewart. Temperature dependence of high field electromechanical coupling in ferroelectric ceramics. Journal of Physics D: Applied Physics, 2010, 43 (16), pp.165404. 10.1088/0022-3727/43/16/165404 . hal-00569584

\section{HAL Id: hal-00569584 \\ https://hal.science/hal-00569584}

Submitted on 25 Feb 2011

HAL is a multi-disciplinary open access archive for the deposit and dissemination of scientific research documents, whether they are published or not. The documents may come from teaching and research institutions in France or abroad, or from public or private research centers.
L'archive ouverte pluridisciplinaire HAL, est destinée au dépôt et à la diffusion de documents scientifiques de niveau recherche, publiés ou non, émanant des établissements d'enseignement et de recherche français ou étrangers, des laboratoires publics ou privés. 


\title{
Temperature dependence of high field electro-mechanical coupling in ferroelectric ceramics
}

\author{
P. M. Weaver, M. G. Cain, M. Stewart \\ National Physical Laboratory, Hampton Road, Teddington, Middlesex, TW11 0LW \\ E-mail: paul.weaver@npl.co.uk
}

\begin{abstract}
A study of the temperature dependence of the electromechanical response of ferroelectric Lead Zirconate Titanate (PZT) ceramics at high electric fields (up to 1.3 $\mathrm{kV} / \mathrm{mm}$ ) is reported. Simultaneous measurements were performed of strain, electric field and polarisation to form a complete response map from room temperature up to 200C. An electrostrictive model is shown to provide an accurate description of the electromechanical response to high levels of induced polarisation and electric field. This provides a method for decoupling strain contributions from thermal expansion and polarisation changes. Direct measurements of electrostriction and thermal expansion, above and below the Curie temperature are reported. Electrostriction coefficients are shown to be temperature dependent in these ceramic materials, with different values above and below the Curie temperature.
\end{abstract}

PACS numbers: 77.80.-e, 77.65.-j, 85.50.-n, 85.80.Jm 


\section{Introduction}

Recent technological interest in piezoelectric materials has been fuelled by new application areas as a component in magneto-electric composites [1] for high density electronic memory [2], highly sensitive magnetic field sensors [2, 3], and for electrical control of magnetization for a range of spintronics applications [4]. Piezoelectric materials are also widely used as actuators, where they continue to find new and extended applications such as injector valves for diesel engines [5], ultra-low power electronic locks and valves [6] for industrial and domestic fluid controls. 'Soft' PZT $\left(\mathrm{Pb} \mathrm{Zr_{x }} \mathrm{Ti}_{1-x} \mathrm{O}_{3}\right)$ ceramics are the materials of choice in many of these applications due to their high piezoelectric coefficients. However, these materials are sensitive to temperature changes with strong coupling between thermal, mechanical and electrically induced polarisation changes.

For data storage applications the ability to write electrically and read magnetically would improve speed and data retention $[7,2]$. In a composite magneto-electric material the coupling is achieved through strain. Piezoelectric strain in response to an applied electric field is indistinguishable from thermally induced strain. This could lead to thermally induced writing and data loss. In sensor applications, it may be possible to partially cancel a.c. thermal noise due to pyroelectric currents [8], but strain-coupled d.c. drift could not be so easily compensated. Careful expansion matching or compensation for the thermal strain in the piezoelectric material would be required to minimize this effect. In actuator applications thermal drift can severely limit the operating range of the electromechanical device. For instance, in a valve, the position of the sealing member relative to the valve seat is a critical parameter in controlling the flow rate. In bender actuators the ceramic is often bonded to a metal substrate, so careful matching to the substrate expansion is required to achieve stable mechanical output across a wide temperature range. In all these applications, a strain reference is fixed either at the bonding stage in the manufacturing process, or with reference to a fixed mechanical point such as the seat of a valve. It is therefore essential to consider the strain output relative to a datum at a particular temperature - a factor that is not usually taken into account in measurements of piezoelectric properties.

In most of these applications large electric fields are needed to maximise energy density. In this high field regime, linear coupling coefficients are of limited value, and the temperature dependency of the non-linear high field electromechanical coupling dominates performance. Many of the fundamental characteristics of ferroelectric materials are defined by the temperature dependency of their properties. Changes in permittivity and spontaneous polarisation around the Curie temperature are central to the characterisation and understanding of these materials and have been well studied [9]. The effects of temperature on the electromechanical properties are less well understood. Data are available on the temperature variation of low field properties such as piezoelectric coefficients obtained from resonance measurements [10]. These are not sufficient to provide information on the high field properties which are dominated 
by non-linear effects, and tell us nothing about thermal drift in the electromechanical performance. Where measurements have been made of large field ferroelectric strain loops [11], these are not related to a datum, so provide no information on the absolute strain. Thermal expansion measurements have been made in PZT ceramics [12, 13], reporting very low values for the thermal expansion coefficient of depoled ceramic (0-2 $\times 10^{-6}{ }^{\circ} \mathrm{C}^{-1}$ ) and negative values for poled ceramics $[12,13]$. Whilst these measurements provide very useful information on phase transitions, they were performed under short circuit conditions, so provide no information on the finite field electromechanical coupling. The need to obtain finite field piezoelectric measurements as a function of temperature relative to a datum has been recognised more recently with regard to the application in diesel injector valves [14]. Direct strain measurements were not reported and the strain relative to the reference was calculated from separate measurements of non-referenced loops, remanent strain, and thermal expansion. The relationship to the polarisation and behaviour above the Curie temperature were not reported.

Thermal expansion in a ferroelectric material is complicated by coupling between the temperature dependent remanent polarisation and the strain. This coupling is influenced by the polarisation induced by an applied electric field, leading to a complicated interdependency between temperature, electric field and strain. This coupling can be understood by studying the electrostrictive properties of the material. It is common to neglect electrostriction when dealing with small field piezoelectric effects on a poled ceramic [15]. In this case the total remanent polarization is not changed significantly by relatively small changes in the induced polarization. High electric fields induce polarisation changes which may be comparable to the remanent polarization. This is particularly significant at high temperatures, but also at more moderate temperatures for soft materials. In these cases the electrostrictive coupling becomes important. The low Curie temperature of soft PZT means that large changes in spontaneous polarization occur over a temperature range encountered in many practical situations, and this electrostrictive coupling to the temperature dependent polarization dominates the thermal expansion and high field electromechanical response.

It can be shown $[16,17]$ that the linear piezoelectric effect can be described in the low field limit as an electrostrictive strain with the polarisation equal to the remanent polarisation. This relationship can be used to estimate piezoelectric coefficients from measurements of remanent polarisation [18] assuming the electrostriction coefficients do not change with temperature. Room temperature measurements of the electrostrictive coefficients have long been available for some ferroelectric materials from resonance techniques, particularly for Barium Titanate $[19,16]$. It is often asserted that the electrostrictive coefficients are independent of temperature, but experimental evidence for this is scant. This is particularly true for PZT. This assertion is based on values obtained indirectly by means of a number of assumptions, or measurements over a restricted temperature range or for single crystals or for materials other than PZT [20].

The objective of the work reported here was to obtain for the first time, a complete mapping of the strain - polarisation - electric field relationship from room temperature 
to above the Curie temperature for soft PZT ceramics. It was a key objective to relate all the strain measurements to a value at a reference temperature so that drift in the actuation position from zero to high electric field could be measured. Measurements were performed for both unipolar and bipolar (switching) cycles. We also provide for the first time electrostriction coefficients obtained from direct measurements of the strain and polarisation, and show that these are temperature dependent above $100^{\circ} \mathrm{C}$.

\section{Experimental Details}

A vertical pushrod dilatometer was used to measure electrically and thermally induced strain. Corrections were made for the pushrod expansion by calibration against a sample of known expansivity. Samples used for the experiments were $25 \mathrm{~mm}$ x $5 \mathrm{~mm}$ x $0.3 \mathrm{~mm}$ sheets of a commercial soft PZT (Fuji Ceramics C91), with approximately $2 \mu \mathrm{m}$ of Ni electrode on both $25 \mathrm{mmx} 5 \mathrm{~mm}$ faces. Strain was measured in the $25 \mathrm{~mm}$ length for an electric field applied across the $0.3 \mathrm{~mm}$ thickness (this was also the direction of poling). This is referred to in this paper as transverse expansion or contraction. Strain in the poling direction is referred to as longitudinal. A positive strain refers to an expansion. Note that results in this paper are mostly plotted as negative strain (noted on the graph labels) to present the familiar direction of the strain loops.

For polarisation measurement a capacitor $(C=17.2 \mu \mathrm{F})$ was placed in series with the piezoelectric element (approximately $17 \mathrm{nF}$ at room temperature). The voltage across the capacitor $(V)$ was measured by means of a high input impedance unity gain instrumentation amplifier. The capacitor was discharged at the start of the experiment. Changes in polarisation from this point were computed from the relation:

$$
\Delta P=\frac{C V}{A}
$$

where $\mathrm{A}$ is the ceramic electrode area. The small amount of drift due to input bias currents was measured and compensated for in the results. Electric field was applied using a high voltage amplifier under computer control. Simultaneous measurements were made of the applied voltage, charge, extension and temperature. Separate experiments were performed for bipolar and unipolar electrical cycles. For the bipolar experiment a loop sequence of linear ramps $0 \mathrm{~V}$ to $+400 \mathrm{~V}$ to $-400 \mathrm{~V}$ to $0 \mathrm{~V}$ was applied over a period of $40 \mathrm{~s}$. Two loops were taken for each temperature point (approximately every $10^{\circ} \mathrm{C}$ ) for a heating/cooling rate of $20^{\circ} \mathrm{C}$ per hour. The unipolar loop sequence was $0 \mathrm{~V}$ to $400 \mathrm{~V}$ to $0 \mathrm{~V}$ over $80 \mathrm{~s}$. The heating/cooling rate for the unipolar experiments was $10^{\circ} \mathrm{C}$ per hour.

\section{Results and Discussion}

Electrical loops recorded at different points across the temperature range are shown in Figure 1. Figure 1(a) shows polarisation - electric field loops. At $19{ }^{\circ} \mathrm{C}$ characteristic ferroelectric loops were observed with a remanent polarisation of approximately $0.3 \mathrm{C}$ 


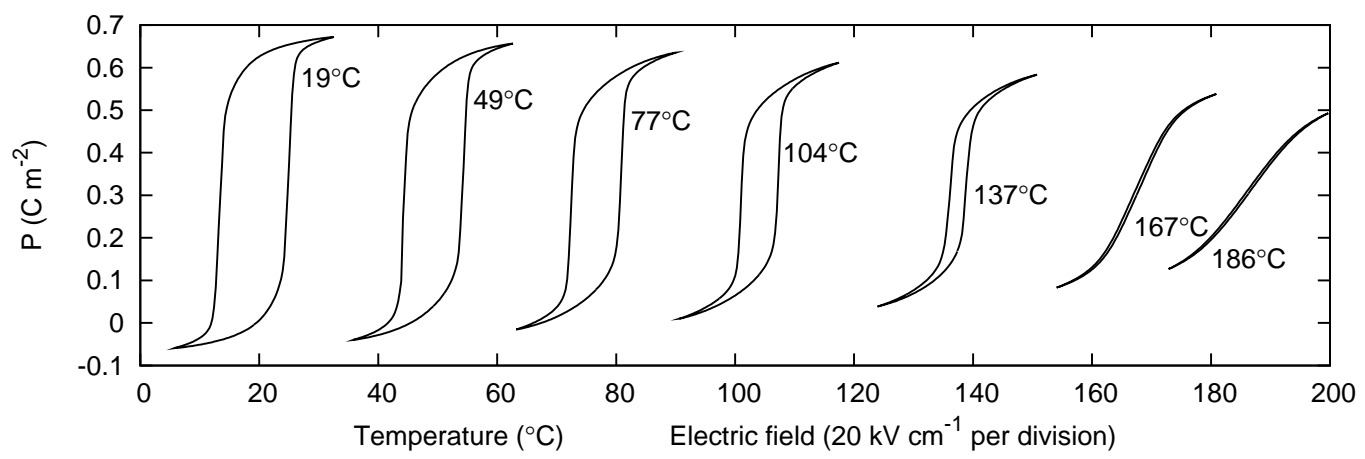

(a)

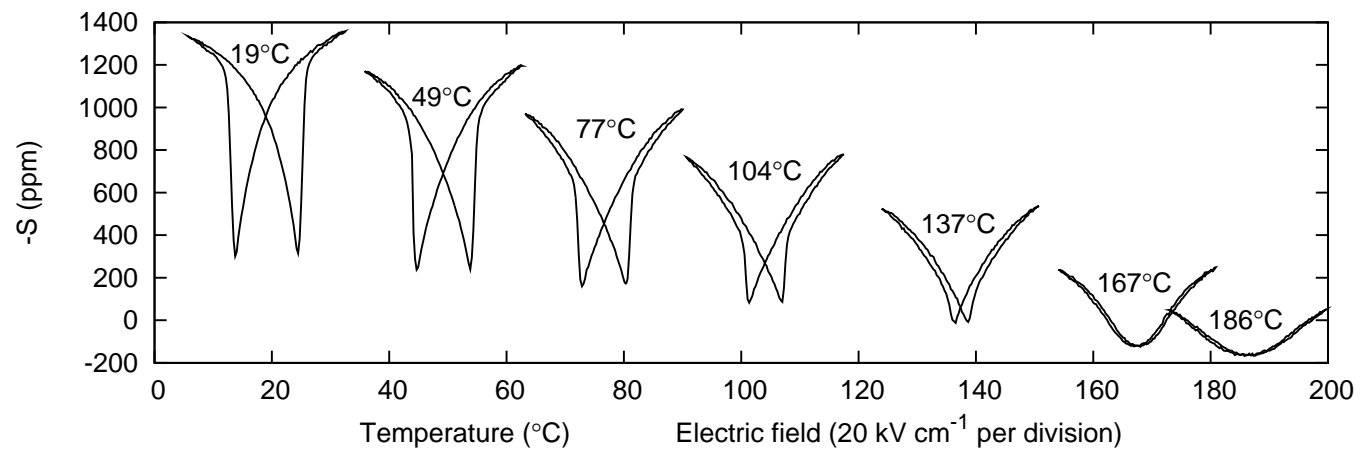

(b)

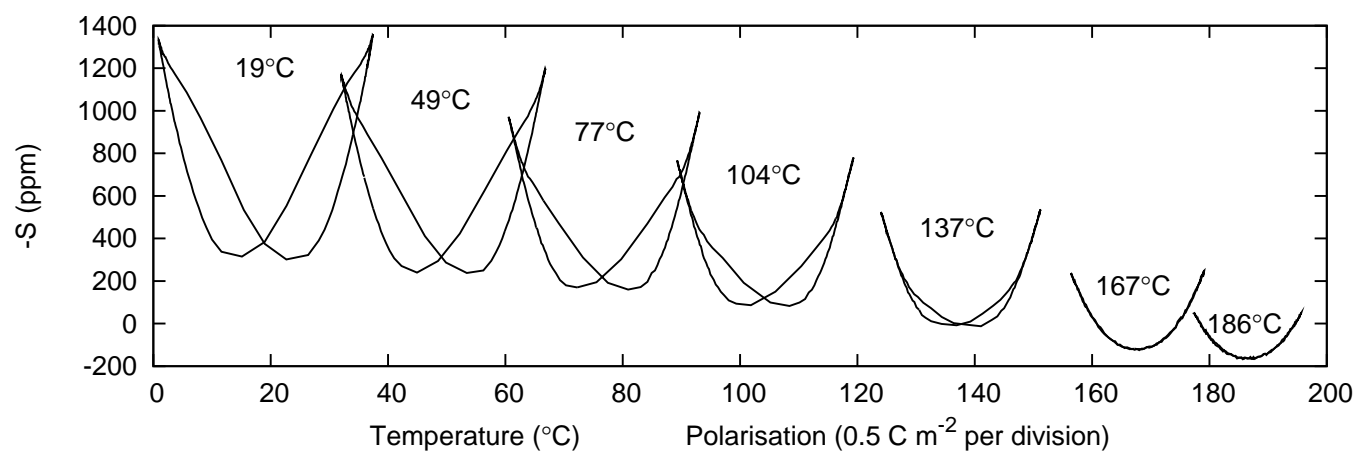

(c)

Figure 1. Composite plots showing bipolar a) polarisation-field, b) strain-field and c) strain-polarisation loops at different temperatures. The $\mathrm{x}$ axis values for each loop (electric field or polarisation) were offset to centre each loop on the temperature at which it was measured. 


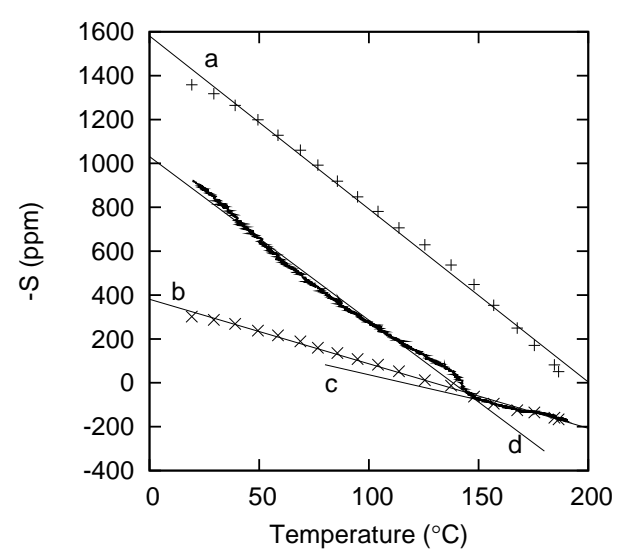

Figure 2. Thermal expansion during temperature cycling with bipolar loops. a is a straight line fit to the loop maxima $(+)$ and $\mathbf{b}$ is a straight line fit to the loop minima $(\times)$. $\mathbf{c}$ is a straight line fit to the remanent strain (solid line) for $T>148^{\circ} \mathrm{C}$ while $\mathbf{d}$ is a fit to the portion of the curve $T<138^{\circ} \mathrm{C}$.

$\mathrm{m}^{-2}$ at room temperature, typical of this type of material [21]. At $186{ }^{\circ} \mathrm{C}$ the hysteresis has disappeared and the dielectric response becomes paralectric, albeit with a high and non-linear permittivity $\left(\epsilon_{r} \approx 15000\right)$. The transition from ferroelectric to paraelectric behaviour occurs approximately between $140{ }^{\circ} \mathrm{C}$ and $150{ }^{\circ} \mathrm{C}$. This will be referred to as the transition temperature. Figure 1(b) shows the strain - field loops. The familiar 'butterfly' loop is observed at the lower temperatures. Above the transition temperature the strain hysteresis also disappears. However, there is still a strong eletromechanical response well above the transition temperature. This is shown in Figure 2 which plots the loop maxima, minima, and the loop end point at $0 \mathrm{~V}$ (the remanent strain). The maxima and minima of the strain loops show no discontinuity or other anomaly on passing through the transition temperature. The remanent strain shows a step change in strain as well as a change in slope at the transition temperature. The remanent strain closely follows the remanent polarisation (see Figure 4) except for a gradual strain drift with increasing temperature corresponding to an underlying thermal expansion. It is not possible to specify a unique expansion coefficient for these loops because it depends on the point in the loop that is measured i.e. the thermal expansion depends on the electrical conditions. For example, the loop maxima and remanent strain show an expansion of approximately $7.5-8.0 \times 10^{-} 6{ }^{\circ} \mathrm{C}^{-1}$ while the loop minima give a value of $2.9 \times 10^{-} 6{ }^{\circ} \mathrm{C}^{-1}$.

Figure 1(c) shows the strain - polarisation loops. Above the transition temperature, these loops become very closely parabolic. This is shown more closely in Figure 3(a) where the experimental data are compared with a parabolic line obtained by a least squares fit (see below for line fitting). The close parabolic relationship is only seen in the strain as a function of polarisation and is characteristic of electrostrictive behaviour [17]. The strain shows a much more complicated dependency on electric field (Figure 1(b)).

Considering only the transverse strain (orthogonal to the poling axis) with the $\infty m$ 


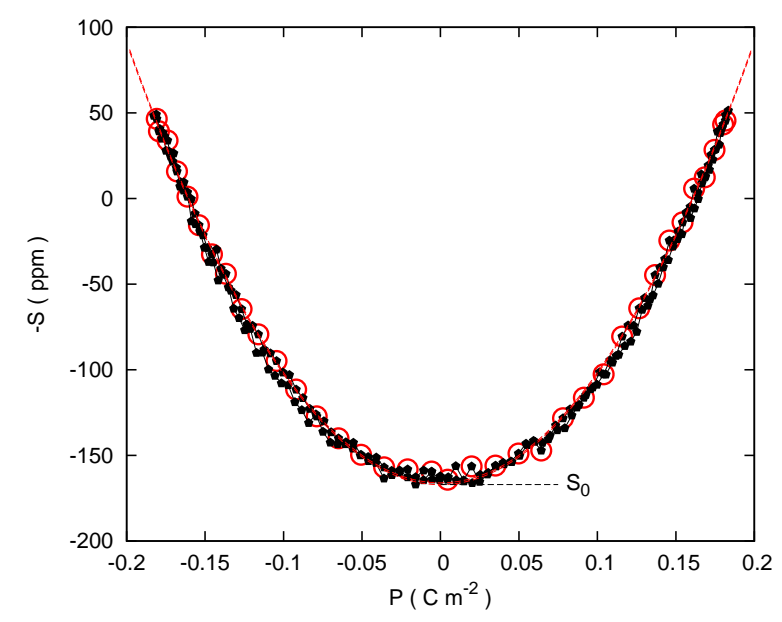

(a)

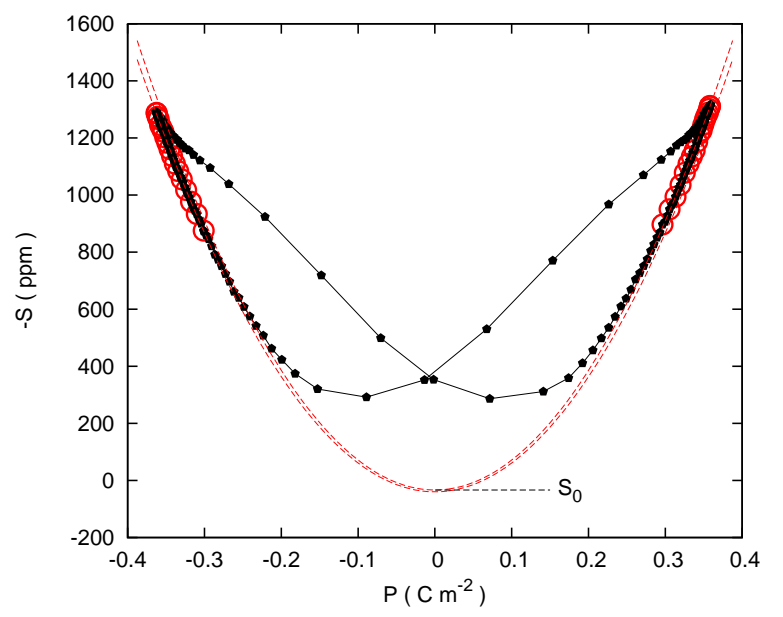

(b)

Figure 3. SP loops at a) $186^{\circ} \mathrm{C}\left(T>T_{C}\right)$ and b) $19^{\circ} \mathrm{C}\left(T<T_{C}\right)$. Dashed lines show the quadratic fit to the points in the stable state region (see discussion), marked as red circles.

point group symmetry of a poled ceramic [22], the electrostriction can be expressed as [17]:

$$
S_{1}=Q_{12} P_{3}^{2}
$$

where $S_{1}$ is the strain, and $P_{3}$ is the polarisation in the direction of the poling axis. $Q_{12}$ is the longitudinal electrostriction coefficient.

The experiment measures changes in polarisation, so the determination of zero polarisation requires some care. Figure 4 shows the values of the polarisation at different points in the loop, and their variation with temperature. The maximum and minimum polarisation diminished gradually with increasing temperature, and showed no anomaly at the transition temperature, approximately tracking the strain maximum. The remanent polarisation decreased somewhat more rapidly, and underwent 


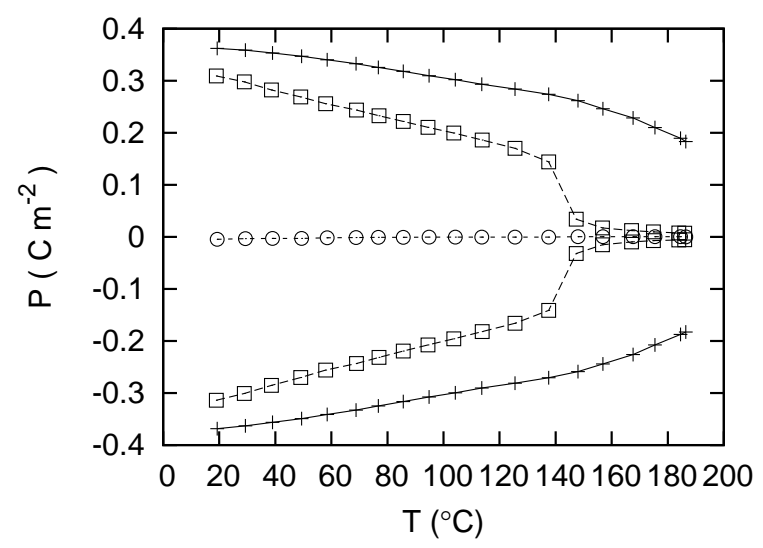

Figure 4. Variation of bipolar PE loop maximum and minimum (+), mean (o) and remanent $(\square)$ polarisation with temperature

a step change at the transition temperature. The polarisation averaged over each loop remained very nearly constant over the temperature range. The average of the positive and negative remanent polarisation was equal to the same value, and also did not vary with temperature. Above the transition temperature, the remanent polarisation approached the same value asymptotically from both directions. The correlation of the different measures of the mean polarisation, and the fact that the remanent polarisation approached the same value above the transition temperature, allows the identification of a single unique value for the polarisation zero. This was set as zero in Figure 4 and all subsequent figures in this paper.

In these experiments the strain varied both with temperature and polarisation and was measured relative to a reference value. Equation 2 must therefore be re-stated more generally as:

$$
S_{1}(T, P)=S_{0}(T)+Q_{12}(T)\left(P_{3}\right)^{2}
$$

where $S_{1}$ is the strain measured from the fixed reference and $S_{0}$ is the strain at zero polarisation at a particular temperature, measured from the same reference. $S_{0}(T)$ therefore represents the thermal expansion of the lattice which is additional to the strain related to polarisation changes. Note that $Q_{12}$ is usually negative reflecting the fact that the striction is negative in the transverse direction, so $S_{1}<S_{0}$ (negative strain $-S$ is the quantity plotted in Figure 1 - Figure 3). $S_{0}$ and $Q_{12}$ can therefore be found from a plot of $S_{1}$ against $P_{3}^{2}$ by linear regression. An excellent fit to the experimental data is obtained above the transition temperature as shown in Figure 3(a). Below the transition temperature, the points in the cycle where the electric field was decreasing from its peak value to zero (open circles in Figure $3(\mathrm{~b})$ ) were found to provide an excellent fit to Equation 3. The electrostriction coefficient, and $S_{0}$ thus obtained are plotted as functions of temperature in Figure 5.

The electrostriction coefficient (Figure $5(\mathrm{a})$ ) is approximately constant up to $100{ }^{\circ} \mathrm{C}$ with a value between $-0.0100 \mathrm{C}^{-2} \mathrm{~m}^{4}$ and $-0.0105 \mathrm{C}^{-2} \mathrm{~m}^{4}$. This is slightly lower than 


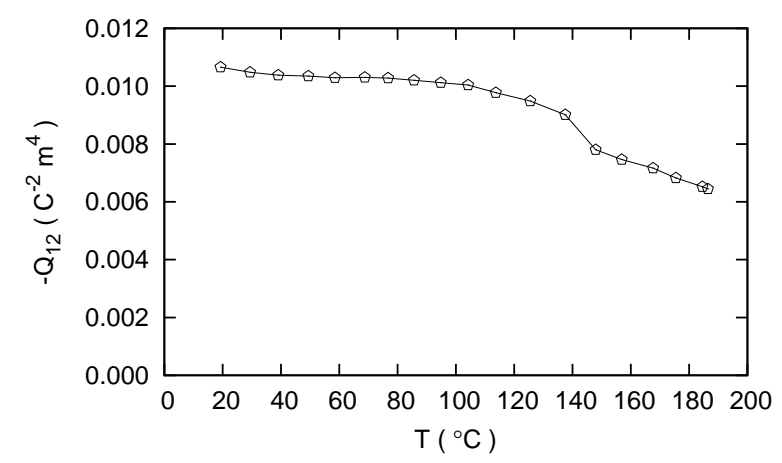

(a)

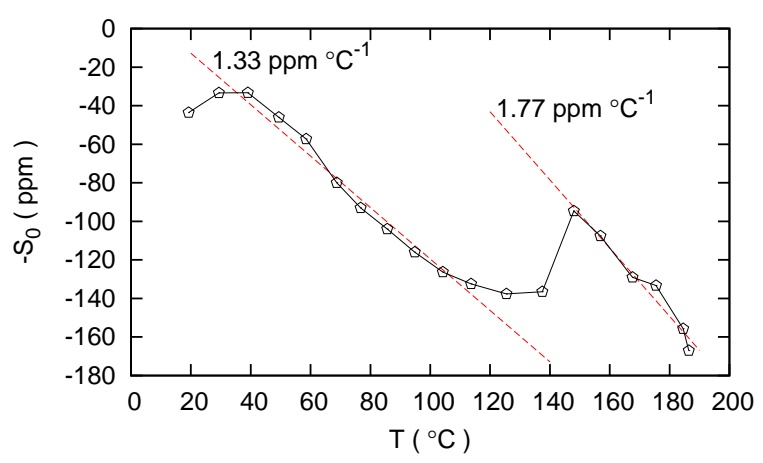

(b)

Figure 5. Electrostriction coefficient, $Q_{12}$, and strain zero $S_{0}$ as functions of temperature (bipolar cycles). In (b) the dashed lines represent straight line fits to the approximately linear portions of the curves.

the value of $-0.0158 \mathrm{C}^{-2} \mathrm{~m}^{4}$ measured by Haun [20] for undoped PZT with $\mathrm{x}(\mathrm{Ti})=0.52$. Above $100{ }^{\circ} \mathrm{C}$ the coefficient drops more rapidly up to the transition temperature. Above the transition temperature the electrostriction coefficient (approximately $0.0075 \mathrm{C}^{-2} \mathrm{~m}^{4}$ ) reduces linearly with increasing temperature. This could be explained by an increasing contribution from $180^{\circ}$ domain wall motion with increasing temperature. Zhang [18] reports that under weak external driving fields the major part of the domain-wall response is generally thought to be from non- $180^{\circ}$ walls and $180^{\circ}$ walls are almost idle. The reason given is that $180^{\circ}$ wall width is much narrower (probably on the order of one unit cell) in comparison with non- $180^{\circ}$ wall which implies that there is a much deeper potential well for $180^{\circ}$ walls. Whilst Zhang's results suggest that there is a larger response from $180^{\circ}$ domain walls in soft PZT, it is still reasonable to expect that $180^{\circ}$ domain walls will become more active as the temperature increases. Because the piezoelectric activity in soft PZT is strongly related to non- $180^{\circ}$ domain wall movements, but a large part of the polarisation response is due to $180^{\circ}$ domain wall movement, the electrostriction coefficient might be expected to vary with temperature in these materials. Increased $180^{\circ}$ domain wall mobility may also explain why the electrostriction coefficient values reported here for soft PZT are somewhat lower than previously reported values for harder compositions. It is interesting to note that 


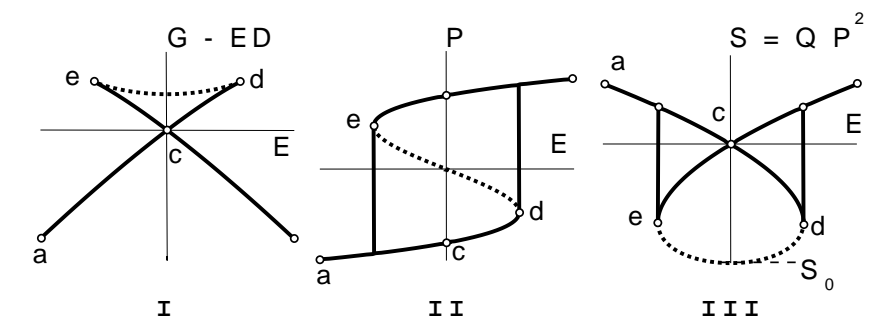

Figure 6. Stability from free energy expansion for $T<T_{c}$. (I) free energy $G_{1}-E D$, (II) polarisation, and (III) strain as functions of electric field. a to $\mathbf{c}$ is stable, $\mathbf{c}$ to $\mathbf{d}$ is metastable.

this process continues even above the transition temperature suggesting that domain processes are still contributing significantly to the dielectric and electromechanical response even well above the transition temperature.

The value of $S_{0}$ thus obtained can be interpreted by consideration of the free energy expansion in the presence of an applied field. The free energy expansion as a function of electric displacement, $D$, is given by [9]:

$$
\begin{aligned}
& G_{1}=\frac{\alpha}{2} D^{2}+\frac{\gamma}{4} D^{4}+\frac{\delta}{6} D^{6} \\
& E=\left(\frac{\partial G_{1}}{\partial D}\right)_{T}=\alpha D+\gamma D^{3}+\delta D^{6}
\end{aligned}
$$

where $\alpha$ varies with temperature, being positive above the Curie temperature, $T_{C}$, and negative below it. The coefficients $\gamma$ and $\delta$ are assumed to be constant and positive corresponding to a second order phase transition. These assumptions are unlikely to be valid for the temperature range of interest here, but this does not affect the qualitative conclusions of this discussion. In the following discussion we use the approximation $D \approx P$ and treat the two terms interchangeably. From Equations 4 and 5 we can evaluate the free energy in the presence of an electric field:

$$
G=G_{1}-E D
$$

At temperatures $T>T_{C}$ there are no states of lower free energy for any value of the electric field (function not plotted). The states described by Equation 6 are therefore stable for all values of the electric field. The free energy function (Equation 6) for $T<T_{C}$ is plotted in Figure 6(I). The stable states (a to $\mathbf{c}$ ) are identified in the same way as for $T>T_{C}$, but now there are metastable states (c to $\mathbf{d}$ ) where a lower free energy exists for the same electric field. An energy barrier separates the metastable from the stable states. The states $\mathbf{d}$ to e are unstable with no energy barrier to the transition. The corresponding points on the PE loop are shown in Figure 6(II). The transition does not occur at zero polarisation, but switches from one polar state to another. The unstable states $\mathbf{d}$ to $\mathbf{e}$ include the state of zero polarisation i.e. the unstable centro-symmetric crystal. Using the electrostrictive relationship (Equation 2) with Equation 6 provides the strain as a function of electric field. The familiar butterfly 
curve is obtained as shown in Figure 6(III) for $T<T_{C}$. The unstable centro-symmetric state corresponds to the strain minimum, $S_{0}$. If the polar switching was the only factor, then $S_{0}$ would not vary with temperature. However, anharmonicity in the vibration of the relatively fixed ionic structure causes an underlying thermal expansion and therefore a change in $S_{0}$. Extrapolation of the electrostrictive curve therefore provides a means of separating temperature dependent polarisation-induced strain from the underlying lattice expansion. The actual response is the sum of this lattice expansion and the electrostriction calculated through equation 3 .

Up to $100{ }^{\circ} \mathrm{C}$ a linear expansion in $S_{0}$ is observed with an effective expansion coefficient of $1.33 \times 10^{-6}{ }^{\circ} \mathrm{C}^{-1}$. Just below the transition temperature the expansion coefficient reduces to a very low value. A contraction is observed in the region of the transition temperature, indicative of a first order crsytallographic phase change. Above the transition, the expansion proceeds linearly with a coefficient of $1.77 \times 10^{-6}{ }^{\circ} \mathrm{C}^{-1}$. These figures are in line with previous measurements for unpoled ceramics $[12,13]$.

For the transverse expansion measurements reported here, changes in the remanent polarisation couple electrostrictively to the strain causing an expansion on heating. This reinforces the underlying thermal expansion of the lattice. If measured in the direction of polarisation, the changes in remanent polarisation would lead to a contraction on heating, which would oppose the underlying lattice expansion. For unpolarised samples, the electrostrictive contraction would be approximately zero, so a weak expansion would be observed. For poled samples, the electrostrictive contraction would dominate leading to negative expansion. Above the Curie temperature there is no remanent polarisation, so zero field strain in both directions reverts to a normal expansion on heating. The transverse measurements reported here therefore help to explain previous reports of negative thermal expansion in poled PZT ceramics [12, 13].

The strain - polarisation curves shown in Figure 7 have been adjusted by applying a temperature dependent offset of $0.8 \times 10^{-6}{ }^{\circ} \mathrm{C}^{-1}$. This is the value of the total expansion across the temperature range shown in Figure $5(\mathrm{~b})$. The value is so low due to the the contraction at the transition temperature. It is used here to compensate the curves to remove the average overall expansion (this figure is arbitrary as discussed below). We observe that all the curves nearly lie on the same parabolic curve, indicating that there is an approximate underlying electrostriction that applies across the temperature range. Very similar results were obtained for both unipolar and bipolar (stable region) cycles. However, the family of curves can not be made to lie on the same curve for any single pair of values of expansion and electrostriction coefficients. This is a reflection of the temperature dependence of these coefficients shown in Figure 5.

These results confirm that the electrostrictive model provides an accurate description of the electro-mechanical response at high levels of induced polarisation and electric field, as long as the system is kept within the stable region of the free energy diagram. This is true even when there is significant non-linearity and hysteresis present in the strain - field response. This implies that the electrostrictive description of the piezoelectric behaviour applies not just to intrinsic crystallographic distortions, but 


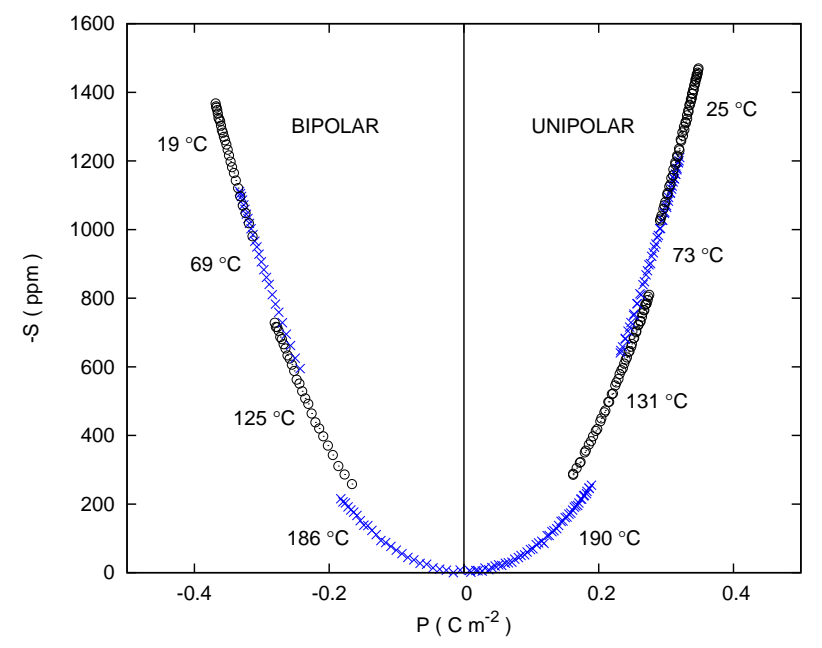

Figure 7. Strain -v- Polarisation compensated for a thermal expansion of $0.8 \times 10^{-6}$ ${ }^{\circ} \mathrm{C}^{-1}$. The left half of the plot shows the stable regions of the bipolar cycles (negative half only shown). The right half of the plot shows unipolar cycles.

also to the changes in strain and polarisation due to extrinsic domain wall motion. The fact that the relationship holds across the temperature range, and the electrostriction coefficient reduces with increasing temperature is a strong indication that both $180^{\circ}$ and non- $180^{\circ}$ domain wall processes are accommodated within this model. However, the model does not apply in the region of metastability where the field is applied against the direction of the remanent polarisation.

\section{Conclusions}

Measurements are reported of the temperature dependent transverse actuation response of PZT ceramics relative to a reference length for large field bipolar and unipolar electrical cycles. Simultaneous measurements were made of the strain, electric field and polarisation to form a complete Strain - Polarisation - Electric Field map from room temperature up to $200{ }^{\circ} \mathrm{C}$. Remanent strain and polarisation show a transition in the region of the Curie temperature, although significant actuation activity is observed well above this transition temperature. The temperature dependent movement range is offset by thermal expansion.

An electrostrictive model provides an accurate description of the device response to high levels of induced polarisation and electric field below, as well as above, the transition temperature as long as the system is kept within the stable region of the free energy diagram. This is true even though there is significant non-linearity and hysteresis in the strain - field response.

Direct measurements of electrostriction coefficients and thermal expansion, above and below the transition temperature are reported. Electrostriction coefficients are shown to be temperature dependent in these ceramic materials, with different values 
above and below the Curie temperature.

\section{Acknowledgements}

The authors would like to acknowledge the support of the Technology Strategy Board and the UK National Measurement Office. We would also like to acknowledge the support of the APAHOE project partners Servocell Ltd., Diameter Ltd., and University of Southampton.

[1] Zhai J, Xing Z, Dong S, Li J and Viehland D 2008 J. Am. Cer. Soc. 91351

[2] J. F. Scott J F 2007 Science 315954

[3] Vopsaroiu M, Blackburn J, Muniz-Piniella A and Cain M G 2008 J. Appl. Phys. 103 07F506

[4] Lottermoser T, Lonkai T, Amann U, Hohlwein D, Ihringer J and Fiebig M 2004 Nature 430541

[5] Mock R and Lubitz K 2008 in Piezoelectricity ed. Heywang W, Lubitz K and Wersing W (Berlin: Springer) Ch.13

[6] Weaver P M and Zheng Y, Proceedings of Actuator 2004, Bremen, pp.426-429, 2004.

[7] Eerenstein W, Mathur N D and Scott J F 2006 Nature 442759

[8] Zhai J, Xing Z, Dong S, Li J and Viehland D 2008 Appl. Phys. Lett. 93072906

[9] Lines M E and Glass A M 1977 Principles and Applications of Ferroelectrics and Related Materials (Oxford: Clarendon) Section 3.3

[10] Sabat R G, Mukherjee B K, Ren W, and Yang G 2007 J. Appl. Phys. 101064111

[11] Maiwa M, Kim S H and Ichinose N 2003 Appl. Phys. Lett. 834396

[12] Cook W R, Berlincourt D A and Scholz F J 1963 J. Appl. Phys. 341392

[13] Seveyrat L, Lemercier M, Guiffard B, Lebrun L, and Guyomar D 2009 Ceram. Int. 3545

[14] Kungl H and Hoffmann M J 2008 Sensors Actuat A-Phys 144328

[15] Kholkin A L, Akdogan E K, Safari A, Chauvy P F, Setter N 2001 J. Appl. Phys. 898066

[16] Kay H F 1955 Rep. Prog. Phys. 18230

[17] Jona F and Shirane G 1962 Ferroelectric Crystals (Oxford: Pergamon) Section IV-5

[18] Zhang Q M, Wang H, Kim N and Cross L E 1994 J. Appl. Phys. 75454

[19] Mason W P 1948 Phys. Rev. 741134

[20] Haun M J, Zhuang Z Q, Furman E, Jang S J and Cross L E 1989 J. Am. Ceram. Soc. 721140

[21] Jaffe B, Cook W R and Jaffe H 1971 Piezoelectric ceramics (Academic Press) (p.147)

[22] Damjanovic D 1998 Rep. Prog. Phys. 611267 